\title{
Urinary TIMP-1 and MMP-2 levels detect the presence of pancreatic malignancies
}

\author{
R Roy ${ }^{1,2}$, D Zurakowski ${ }^{2,3}$, J Wischhusen ${ }^{1}$, C Frauenhoffer ${ }^{4}$, S Hooshmand ${ }^{4}$, M Kulke ${ }^{2,4}$ and M A Moses ${ }^{\star}, 1,2$ \\ ${ }^{1}$ The Program in Vascular Biology and Department of Surgery, Boston Children's Hospital, Boston, MA, USA; ${ }^{2}$ Harvard Medical \\ School, Boston, MA, USA; ${ }^{3}$ Department of Anesthesia, Boston Children's Hospital, Boston, MA, USA and ${ }^{4}$ Department of Medical \\ Oncology, Dana-Farber Cancer Institute, Boston, MA, USA
}

Background: A majority of patients with pancreatic malignancies, including both pancreatic ductal adenocarcinoma (PDAC) and pancreatic neuroendocrine tumours (pNETs), present with advanced disease due to a lack of specific symptoms and current diagnostic limitations, making this disease extremely difficult to detect. Our goal was to determine whether urinary matrix metalloproteases (uMMPs) and/or their endogenous inhibitors, urinary tissue inhibitor of metalloproteases (uTIMPs), could be detected in the urine of patients with pancreatic malignancies and whether they may serve as independent predictors of disease status.

Methods: Retrospective analyses of urine samples $(n=139)$ from PDAC and PNET patients as well as age- and sex-matched controls were conducted. Urinary MMP-2 and UTIMP-1 levels were determined using ELISA and zymography. Biomarker expression in tumour and normal pancreatic tissues was analysed via immunohistochemistry (IHC).

Results: Multivariable logistic regression analyses indicated that, when controlling for age and sex, uMMP-2 $(P<0.0001)$ and UTIMP-1 $(P<0.0001)$ but not UMMP-9, were significant independent predictors for distinguishing between PDAC patients and healthy controls. Our data also indicated that UMMP-2 was an independent predictor of the presence of pNET. In addition, UTIMP1 levels could differentiate the two cancer groups, PDAC and pNET, respectively. Immunohistochemistry analysis confirmed that MMP-2 and TIMP-1 protein expression is significantly upregulated in PDAC tissue compared with the normal pancreas.

Conclusions: Taken together, our results suggest that the detection of UMMP-2 and UTIMP-1 may have diagnostic value in the detection of pancreatic malignancies and that UTIMP-1 may be useful in distinguishing between pancreatic adenocarcinoma and neuroendocrine tumours.

Pancreatic malignancies are generally subcategorised as either pancreatic ductal adenocarcinoma (PDAC) or pancreatic neuroendocrine tumours (pNETs). Pancreatic adenocarcinoma accounts for over $90 \%$ of pancreatic malignancies. A diagnosis of PDAC has an extremely poor prognosis, with an overall 5-year relative survival rate of only $\sim 5 \%$ and a median survival of $<6$ months (Maitra and Hruban, 2008). Pancreatic neuroendocrine tumours are less common than PDAC and have a more favourable prognosis, although when diagnosed at a late stage this disease is almost always fatal, with an estimated median survival duration of 2 years (Yao et al, 2008). Surgical resection is the only curative therapy for either PDAC or pNET, unfortunately for both diagnoses the majority of patients present with unresectable disease (Li et al, 2004).

Neoplasms of the pancreas are grouped according to their localisation in the pancreas (head, body or tail) or according to the type of cell from which the cancer originated (exocrine or endocrine). Exocrine PDACs are the most common type of pancreatic tumours and account for approximately $90 \%$ of diagnosed pancreatic cancers (Kaur et al, 2012). Neuroendocrine tumours (NETs) are rarer and are characterised by the secretion of hormones and are typically named after the hormone they produce (e.g., gastrinomas, insulinomas and glucagonomas). Pancreatic NETs have a low prevalence in the population of about 1 per 
100000 representing $\sim 1-2 \%$ of all pancreatic neoplasms (Plockinger et al, 2004). In the United States, the incidence and prevalence of pNET has substantially increased in the last three decades (Modlin et al, 2008).

A lack of specific symptoms make both PDAC and pNET difficult to diagnose at an early stage. Currently used blood-based tumour biomarkers are inadequate for either screening or diagnosis. CA19-9 is the only FDA-approved clinical biomarker in use for diagnosis as well as for assessing therapeutic efficacy of PDAC (Steinberg, 1990; Goggins, 2005). CA19-9 has a moderate sensitivity $(\sim 80 \%)$ and specificity $(\sim 60-70 \%)$ for detecting PDAC (Yeo et al, 2002; Ozkan et al, 2003), and only $65 \%$ of patients presenting with resectable pancreatic tumours have elevated CA19-9 levels (Goggins, 2005). CA19-9 levels can be elevated in patients with various benign pancreatobiliary disorders or other non-pancreatic malignancies. For example, upregulation of CA19-9 expression in several cancers (gastric, colon and ovarian) as well as benign conditions (liver cirrhosis, pancreatitis, obstructive jaundice and gastric ulcer) can lead to increased falsepositive results (Ballehaninna and Chamberlain, 2012), whereas a Lewis-negative genotype (Tempero et al, 1987) can lead to falsenegative results, thereby significantly limiting the efficacy of CA199 in PDAC detection and management. Importantly, for the detection of smaller pancreatic tumours, which would represent a better chance of curative resection and significantly improved patient survival, serum CA19-9 has a very low sensitivity ( $\sim 55 \%)$ (Steinberg, 1990; Goggins, 2007). Based on these limitations, the National Academy of Clinical Biochemistry guidelines for PDAC tumour markers in the clinic recommend a limited role for this marker in combination with pancreatic imaging techniques (Goggins, 2007) for the diagnosis of PDAC as well as for therapeutic assessment. Currently available imaging techniques to detect PDAC are also somewhat limited, although endoscopic ultrasound has shown some promise in screening patients at a high risk for PDAC (Canto et al, 2006; Poley et al, 2009).

Two tumour markers currently in clinical use for pNETs are neuron-specific enolase and chromogranin A (CgA) (Seregni et al, 2001; Yao et al, 2011; Ter-Minassian et al, 2013). Of these, CgA, which represents a constitutive neuroendocrine secretory protein, is the most widely accepted biomarker for pNET diagnosis (86\% specificity/69\% sensitivity; Seregni et al, 2001) and CgA levels were found to correlate with disease status in patients with advanced disease, particularly in patients with liver metastases. However, the use of $\mathrm{CgA}$ for pNET management has obvious biological limitations. Elevated $\mathrm{CgA}$ levels are found in cardiac, endocrine and inflammatory diseases, thereby significantly reducing specificity for pNETs (Modlin et al, 2010). In addition, although early decreases in CgA have been associated with favourable outcomes (Modlin et al, 2010), the prognostic and predictive value of CgA has not yet been widely validated and may also be therapy dependent (Kulke et al, 2011). Finally, 25\% NETs do not express CgA resulting in reduced sensitivity for this marker (Zatelli et al, 2007). Therefore, there is an unmet need for novel biomarkers with improved clinical efficacy for pNET.

Several studies have evaluated differentially expressed biomarkers for PDAC in tissue, blood or exocrine pancreatic secretions. In recent years, numerous studies have focused on the discovery of serum-based biomarkers for the early detection of pancreatic cancer (reviewed in Faca et al, 2008; Roy et al, 2010; Sun et al, 2011; Winter et al, 2012), however, thus far, none of these have been successfully adopted in the clinic. Studies using urine as a source of biomarkers for PDAC have been rare (Nolen and Lokshin, 2011). To date, one study has reported that elevated levels of the enzyme urokinase-type plasminogen activator receptor in urine of PDAC patients could identify a clinically high-risk subset of this disease (Sorio et al, 2011). Studies evaluating novel biomarkers in pNET are similarly limited. The goal of our study is to establish a sensitive and specific non-invasive method for the detection of pancreatic malignancies. We have evaluated whether urinary matrix metalloprotease (MMP) or tissue inhibitor of matrix metalloprotease (TIMP) levels may be useful for the detection of PDAC or pNET.

The malignant nature of pancreatic cancer represents an aggressive phenotype characterised by early local invasion and metastasis (Evans et al, 1997; Yang et al, 2001) facilitated by the degradation of type IV collagen and proteoglycans in the basement membrane, as well as changes in expression of MMPs and their endogenous inhibitors TIMPs (Bramhall et al, 1996; Matsuyama et al, 2002). In particular, MMP-2 and MMP-9 are known to facilitate pancreatic tumour progression and aggressiveness of disease (Evans et al, 1997; Matsuyama et al, 2002; Durlik and Gardian, 2012).

We have analysed urine from patients with PDAC, pNET and healthy controls to determine whether the gelatinases MMP-2 and MMP-9 as well as their endogenous inhibitors, the TIMPs, can serve as predictors of disease status. Our results indicate that several MMP species can be detected with significantly higher frequency in urine from pancreatic cancer patients compared with healthy controls. Of these, urinary MMP-2 (uMMP-2) but not urinary MMP-9 (uMMP-9) was a significant independent predictor for distinguishing patients with either PDAC or pNET from controls. In addition, uMMP-2 was an independent predictor of the presence of neuroendocrine cancer. Urinary TIMP-1 (uTIMP-1) levels could also distinguish not only pancreatic malignancies from control but also PDAC from pNET groups. In agreement with these findings, we detected increased expression of MMP-2 and TIMP-1 protein expression in pancreatic tumours compared with normal pancreatic tissues.

\section{MATERIALS AND METHODS}

Urine collection and processing. One hundred thirty-nine samples $(n=139)$ were analysed in this study, including samples from patients diagnosed with PDAC $(n=51)$ and pNET $(n=28)$ and sex-matched controls $(n=60)$. Power analyses were conducted to determine the appropriate numbers of cancer patients and controls, based on comparing the medians and interquartile ranges of the urinary biomarker levels (measured via ELISA) between the study groups using the Mann-Whitney $U$-test. These indicated that to detect a $30 \%$ difference in the median levels $\left(\mathrm{ng} \mathrm{ml}^{-1}\right)$, a minimum sample size of 28 patients in each of the three study groups (Controls, pNET and PDAC) would provide $80 \%$ statistical power using a Bonferroni-adjusted $P$-value to reduce the false discovery rate and assuming that $75 \%$ of the controls would have a lower uMMP value than cancer patients. In our study, we had the benefit of having more PDAC samples $(n=51)$ than required based on the sample size requirements and decided to include 60 controls to provide greater assurance that elevated uMMP biomarkers in pNET or PDAC groups compared with the controls would reflect clinically important differences in the population. Urine was collected according to the institutional bioethical guidelines pertaining to discarded clinical material as previously described (Moses et al, 1998; Roy et al, 2004; Smith et al, 2007; Pories et al, 2008; Roy et al, 2008; Smith et al, 2008). Samples were collected in sterile containers and immediately frozen at $-20^{\circ} \mathrm{C}$. Urine was tested for presence of blood and leukocytes using Multistix 9 Urinalysis Strips (Bayer, Elkhart, IN, USA) and samples containing blood or leukocytes were excluded from analysis.

Substrate gel electrophoresis. Urinary MMPs and their complexes in urine were detected using gelatin zymography as described previously (Moses et al, 1998; Roy et al, 2008). Briefly, urine $(40 \mu \mathrm{l})$ from controls or cancer patients and pure $(2 \mathrm{ng})$ MMP-2 or MMP-9 were mixed with sample buffer (4\% SDS, $0.15 \mathrm{moll}^{-1}$ Tris ( $\left.\mathrm{pH} 6.8\right), 20 \%(\mathrm{v} / \mathrm{v})$ glycerol and $0.5 \%(\mathrm{w} / \mathrm{v})$ 
bromophenol blue), applied without boiling to a 10\% SDSacrylamide gel containing $0.1 \%(\mathrm{w} / \mathrm{v})$ gelatin (Bio-Rad, Hercules, CA, USA) and resolved via electrophoresis. Subsequently, gels were soaked in $2.5 \%$ Triton X-100 with gentle shaking for $30 \mathrm{~min}$. Substrate digestion was conducted by incubating the gels overnight at $37^{\circ} \mathrm{C}$ in substrate buffer $\left(50 \mathrm{mmoll}^{-1}\right.$ Tris-HCL (pH 8), $5 \mathrm{mmoll}^{-1} \mathrm{CaCl}_{2}$ and $0.02 \% \mathrm{NaN}_{3}$ ) as previously described (Moses et al, 1998; Roy et al, 2008). Gels were stained with $0.5 \%$ Coomassie blue R-250 and imaged using Bio-Rad Imager. Bands of enzyme activity were detected as zones of clearance on a background of uniform blue staining. Zymograms were scored independently in a binary manner (presence or absence of MMP expression) by two investigators in a blinded manner.

ELISA. ELISAs (Quantikine kits; R\&D Systems, Inc., Minneapolis, MN, USA) were used to quantify levels of uMMP-2, uMMP-9, uTIMP-1 and uTIMP-2 as previously reported (Smith et al, 2007, 2008). Specimens, standards and reagents were prepared according to the manufacturer's instructions. Protein concentration in urine was determined by the Bradford method using bovine serum albumin as the standard as previously described by us (Moses et al, 1998; Roy et al, 2004; Pories et al, 2008; Roy et al, 2008).

Immunohistochemical analysis. Immunohistochemical (IHC) analysis of human pancreatic cancer tissue arrays (PA483a, T143; US Biomax, Rockville, MD, USA) was conducted using monospecific antibodies against MMP-2 (MAB13405; EMD Millipore, Temecula, CA, USA) and TIMP-1 (E3360; Spring Biosciences, Pleasanton, CA, USA) as described previously (Roy et al, 2011).

Statistical analysis. The nonparametric Mann-Whitney $U$-test was applied to compare medians and interquartile ranges for uMMP-2 and uTIMP-1 levels between study groups. Regression analysis was applied to assess whether group differences in biomarker levels remained significant after adjustment for age. Pearson $\chi^{2}$ test was used to compare percentage of positive MMP expression. Receiver operating characteristic (ROC) curve analysis was used to determine area under the curve and the Youden index was used to identify the optimal threshold cutoff value to maximise classification of PDAC patients and controls (Zhou et al, 2011). Multivariable logistic regression was performed to determine independent predictors for differentiating between PDAC and controls with the likelihood ratio test to assess significance, and predicted probabilities and 95\% confidence intervals (95\% CI) calculated for combinations of the significant independent predictive biomarkers. Our predictive algorithm was based on multivariate logistic regression modelling to derive predicted probabilities of PDAC based on the significant independent urinary biomarkers (uMMP-2 and uTIMP-1) with cutoff values for those biomarkers determined using the Youden index in ROC analysis to maximise sensitivity and specificity of prediction. In addition, $90 \%$ CIs around the predicted probability of PDAC were determined in order to show the possible range that could occur based on each of the four variant combinations in the algorithm. Statistical analysis was conducted using IBM SPSS Statistics (version 21.0, IBM, Armonk, NY, USA). Two-tailed Bonferronicorrected values of $P<0.01$ were considered statistically significant to account for multiple group comparisons (Zhou et al, 2011). Consequently, $P$-values $<0.05$ but not $<0.01$, which may easily reflect type I errors, were not regarded as statistically significant to protect against false discovery.

\section{RESULTS}

Urinary MMP expression is higher in PDAC patients. We initially measured uMMP expression as well as determined which of the distinct MMP species are represented in urine of patients with pancreatic malignancies. Urine from PDAC and pNET patients and controls were subjected to substrate gel electrophoresis and blindly scored for the presence or absence of enzyme activity for multiple MMP species including MMP-2 $(\sim 68 \mathrm{kDa})$ and MMP-9 monomer $(\sim 92 \mathrm{kDa})$, MMP-9 dimer $(\sim 180 \mathrm{kDa})$ and MMP-9/NGAL complex ( $\sim 140 \mathrm{kDa})$ (Figure 1A). Identities of these uMMP species have been previously confirmed and reported by us (Moses et al, 1998; Roy et al, 2008). The percentage of patients with positive expression of uMMPs was calculated and compared for each MMP species between cancer and control groups. Based on univariate analysis, we found that MMPs can be detected in the urine of PDAC and pNET patients with significantly higher frequency than in healthy controls (Figure 1B). Urinary MMP-2 activity was detected with significantly higher frequency in urine of PDAC $(P<0.001 ; 77 \%)$, and pNET patients $(P<0.001 ; 85 \%)$ compared with healthy controls (28\%). Similarly, uMMP-9 monomer ( $\sim 92 \mathrm{kDa}$ species) activity
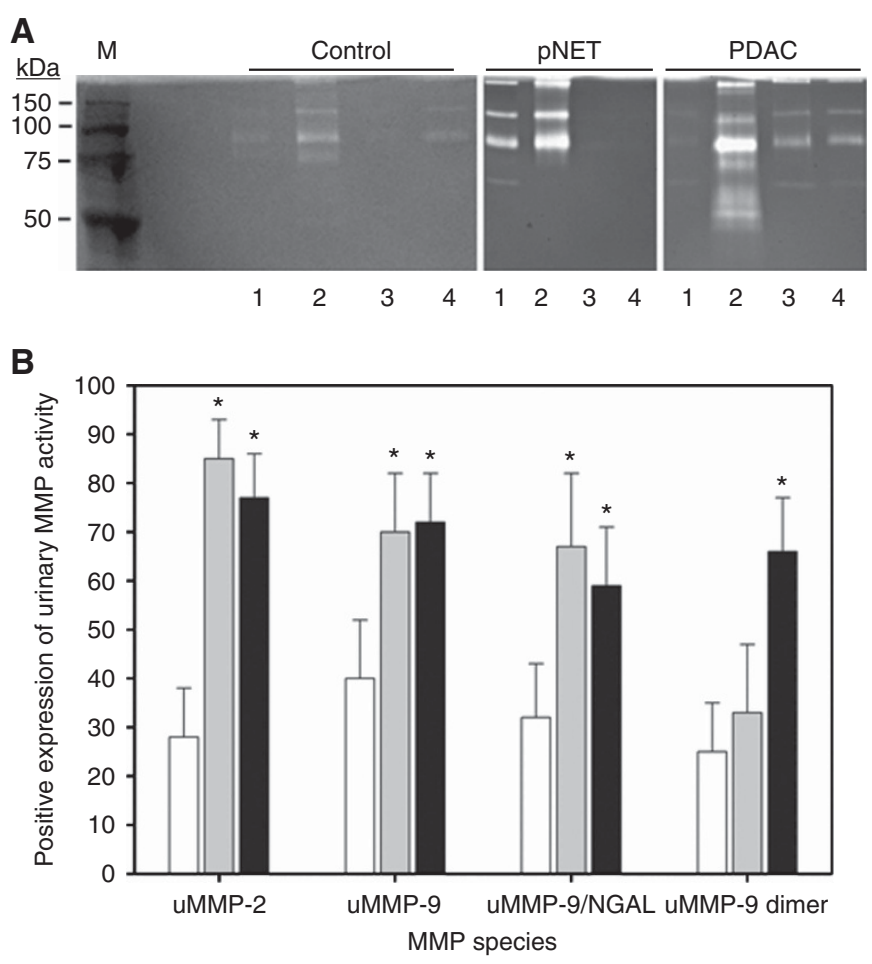

Figure 1. Urinary MMP expression is elevated in samples from PDAC and pNET patients. Representative urine samples (1-4) from Control and PNET and PDAC cancer patients analysed via substrate gel electrophoresis (zymography) with gelatin as the substrate (A). Multiple MMP species were detected including MMP-2 ( 68 kDa) and MMP-9 monomer ( $~ 92 \mathrm{kDa})$, MMP-9 dimer ( $180 \mathrm{kDa})$ and MMP-9/NGAL complex ( $\sim 140 \mathrm{kDa})$. Molecular weight markers are indicated.

Percentages of patients with positive MMP expression for each of the three study groups for uMMP-2, uMMP-9, uMMP-9/NGAL complex, and uMMP-9 dimer (B). Controls (white bar), pNET (grey bar) and PDAC (black bar). Asterisks denote significantly higher percentages of patients in PNET and PDAC groups versus controls (all $P<0.01$ ) with respect to uMMP-2, uMMP-9 and UMMP-9/NGAL. Percentage of PDAC patients with positive expression for UMMP-9 dimer was significantly higher than controls $(P<0.01)$ or pNET $(P<0.01)$ with no differences between pNET vs controls $(P=0.45)$. The error bars represent $95 \% \mathrm{Cls}$ around the observed proportions to provide the precision of the activity data for the four UMMPs in each of the three groups. For each uMMP, Pearson $\chi^{2}$ was applied to assess overall group differences in percentages with positive expression with PDAC and PNET patients compared with healthy controls using Fisher's exact test. 
was detected in $72 \%$ of PDAC and $70 \%$ of pNET patients, respectively, but at much lower frequency in urine from controls (40\%). The uMMP-9 dimer species $(\sim 180 \mathrm{kDa})$ was detected with significantly higher frequency only in PDAC $(P<0.001 ; 66 \%)$ urines compared with pNET $(33 \%)$ or controls $(25 \%)$.

Urinary MMP-2 and TIMP-1 levels can differentiate PDAC patients from normal controls. Based on our findings that uMMP-2 and uMMP-9 activity was detected with significantly higher frequency in pancreatic cancer patients as compared with the controls, we analysed MMP-2, MMP-9 as well as their endogenous inhibitors TIMP-1 and TIMP-2 levels in urine from pancreatic cancer patients and age- and sex-matched controls using ELISAs. Urinary MMP-2 levels were significantly higher in both PDAC (median (interquartile range); $1.2(1.0-1.6) \mathrm{ng} \mathrm{ml}^{-1}$, $n=51, P<0.001)$ and pNET tumour $\left(1.7(1.1-3.3) \mathrm{ng} \mathrm{ml}^{-1}, n=28\right.$, $P<0.001)$ cancer groups compared with the controls $(0.8(0.2-$ 1.0) $\mathrm{ng} \mathrm{ml}^{-1}, n=60$; Table 1). Univariate analysis indicated that while uMMP-2 levels were significantly different between the cancer and control groups, MMP-2 levels alone did not differentiate between PDAC and pNET cohorts $(P=0.04$; Table 1). Indeed, $60 \%$ of the PDAC and $75 \%$ of the pNET cohorts had uMMP-2 values above the 95th percentile of healthy controls $\left(\sim 0.8 \mathrm{ng} \mathrm{ml}^{-1}\right)$, whereas only $40 \%$ had uMMP-2 levels below that respectively. Urinary MMP-9 levels did not differ significantly between the cancer and/or the control groups (Table 1).

Urinary TIMP-1 levels were significantly higher for the PDAC group (median (interquartile range); 4.23 (2.53-13.32) $\mathrm{ng} \mathrm{ml}^{-1}$, $n=51, P<0.001)$ compared with both the pNET $(0.67(0.14-$ 4.30) $\left.\mathrm{ng} \mathrm{ml}^{-1} ; n=28, P<0.001\right)$ and the healthy control groups (0.33 (0.12-2.98) $\left.\mathrm{ng} \mathrm{ml}^{-1} ; n=60\right)$, respectively (Table 1$)$. Sixty-six percent of the PDAC patients had uTIMP-1 levels of $\geqslant 2.98 \mathrm{ng} \mathrm{ml}^{-}$

${ }^{1}$ (the 95th percentile of healthy controls), whereas $<25 \%$ of the pNET patients had higher uTIMP-1 levels. This pattern of uTIMP1 expression was maintained when values were normalised for total urinary protein, with significantly higher uTIMP-1 levels detected in the PDAC cohort (median (interquartile range); 403.2 (156697) $\mathrm{ng} \mathrm{mg}^{-1}$ ) as compared with the pNET (9.4 (4.6-37.7) $\mathrm{ng} \mathrm{mg}^{-1}$ ) or control (13.8 (4.0-74.8) $\mathrm{ng} \mathrm{mg}^{-1}$ ) groups (data not shown). A subset of samples from each group was analysed via immunoblot to confirm the detection of uTIMP-1 ( $\sim 29 \mathrm{kDa}$; data not shown). We also measured uTIMP-2 in urine samples via ELISA, however, uTIMP-2 levels did not differ significantly between the three groups (data not shown).

Individual statistical analyses of each of these markers using logistic regression indicated that while uMMP-2 can distinguish between cancer groups (PDAC and pNET) and controls, uTIMP-1 levels can distinguish not only between PDAC and controls but also between the PDAC and pNET cancer groups (Table 1). Multivariable regression analysis confirmed that uMMP-2 was significantly elevated in patients with PDAC compared with the controls independent of age $(P=0.005)$. Similarly, for uTIMP-1, after controlling for age, regression analysis showed that the difference between PDAC and controls remain statistically significant $(P=0.01)$. With respect to pNET, higher levels of uMMP-2 were observed compared with the controls independent of age $(P<0.001)$. For each significant independent predictive biomarker and their combination, ROC analysis was applied to determine area under the curve (AUC) treating each biomarker as a continuous variable (Figure 2; Table 2). In addition, optimal cutoff values were chosen and based on these cutoff values, sensitivity and specificity was calculated for the respective biomarker. As shown in Table 2, uMMP-2 demonstrated a sensitivity of $70 \%$ (36 of 51) for correctly classifying PDAC patients, whereas uTIMP-1 had a sensitivity of $90 \%$ (46 of 51). The combination of UMMP-2 and uTIMP-1 was associated with a sensitivity of $\sim 91 \%$. Conversely, specificity was $85 \%$ for uMMP-2 (51 of 60 ) and 70\% for uTIMP-1 (42 of 60), respectively, suggesting ability of these biomarkers for correctly classifying controls (Table 2). The optimal cutoff values calculated based on the Youden index were $\geqslant 1.1 \mathrm{ng} \mathrm{ml}^{-1}$ for uMMP-2, which showed the highest specificity, and $\geqslant 0.9 \mathrm{ng} \mathrm{ml}^{-1}$ for uTIMP-1, which showed the highest sensitivity, respectively (Figure 2). The AUC for each of the two predictive biomarkers indicated excellent diagnostic accuracy (Table 2).

Using the chosen cutoff values for UMMP-2 and uTIMP-1, which maximised sensitivity and specificity, logistic regression was utilised to derive a predictive algorithm (Table 3). The four combinations shown in Table 3 are expressed in terms of probability of PDAC and range from a predicted probability of $8 \%$ for a patient with uMMP-2 and uTIMP-1 levels below the cutoff (95\% CI: $3-18 \%$ ) to $85 \%$ for an individual whose uMMP-2 and uTIMP-1 levels are above the cutoff (95\% CI: 72-93\%).

TIMP-1 and MMP-2 are upregulated in pancreatic tumour tissues. In order to determine the expression patterns of TIMP-1 and MMP-2 in human pancreatic cancer tissues, we conducted an analysis of arrays of malignant human pancreatic tumours and matched adjacent normal tissue via IHC (Figure 3). Tissue inhibitor of metalloprotease- 1 protein expression was absent in normal pancreatic tissues ( $0 \%$ positive, $n=16)$ with the exception of pancreatic islets cells, which stained focally positive (Figures $3 \mathrm{~A}$ and $\mathrm{B})$, but TIMP-1 staining frequency was higher in PDAC tissue (97.5\% positive, $n=44, \quad P<0.00001$ ) (Figures $3 \mathrm{C}$ and $\mathrm{D}$ ). Pancreatic islet cells have previously been reported to express high levels of TIMP-1 (Crnogorac-Jurcevic et al, 2002). In our study, the

Table 1. Urinary MMP-2 and TIMP-1 levels can differentiate controls from pancreatic malignancies

\begin{tabular}{|c|c|c|c|c|c|c|}
\hline Variable & Controls $(n=60)$ & pNET $(n=28)$ & $\operatorname{PDAC}(n=51)$ & $\begin{array}{c}\text { PNET vs control } \\
P \text {-value }\end{array}$ & $\begin{array}{c}\text { PDAC vs control } \\
P \text {-value }\end{array}$ & $\begin{array}{c}\text { PDAC vs pNET } \\
P \text {-value }\end{array}$ \\
\hline $\begin{array}{l}\text { Gender } \\
\text { Male } \\
\text { Female }\end{array}$ & $\begin{array}{l}34(57 \%) \\
26(43 \%)\end{array}$ & $\begin{array}{r}20(70 \%) \\
8(30 \%)\end{array}$ & $\begin{array}{l}30(59 \%) \\
21(41 \%)\end{array}$ & 0.24 & 0.85 & 0.33 \\
\hline Age, years & $42 \pm 11$ & $56 \pm 10$ & $61 \pm 11$ & $<0.001^{a}$ & $<0.001^{a}$ & 0.04 \\
\hline uMMP-2, $\mathrm{ng} \mathrm{ml}^{-1}$ & $0.8(0.2-1.0)$ & $1.7(1.1-3.3)$ & $1.2(1.0-1.6)$ & $<0.001^{a}$ & $<0.001^{a}$ & 0.04 \\
\hline uMMP-9, $\mathrm{ng} \mathrm{ml}^{-1}$ & $0.07(0.00-0.14)$ & $0.11(0.07-0.15)$ & $0.04(0.00-0.09)$ & 0.29 & 0.31 & 0.02 \\
\hline uTIMP-1, $\mathrm{ng} \mathrm{ml}^{-1}$ & $0.33(0.12-2.98)$ & $0.67(0.14-4.30)$ & $4.23(2.53-13.32)$ & 0.66 & $<0.001^{a}$ & $<0.001^{\mathrm{a}}$ \\
\hline
\end{tabular}




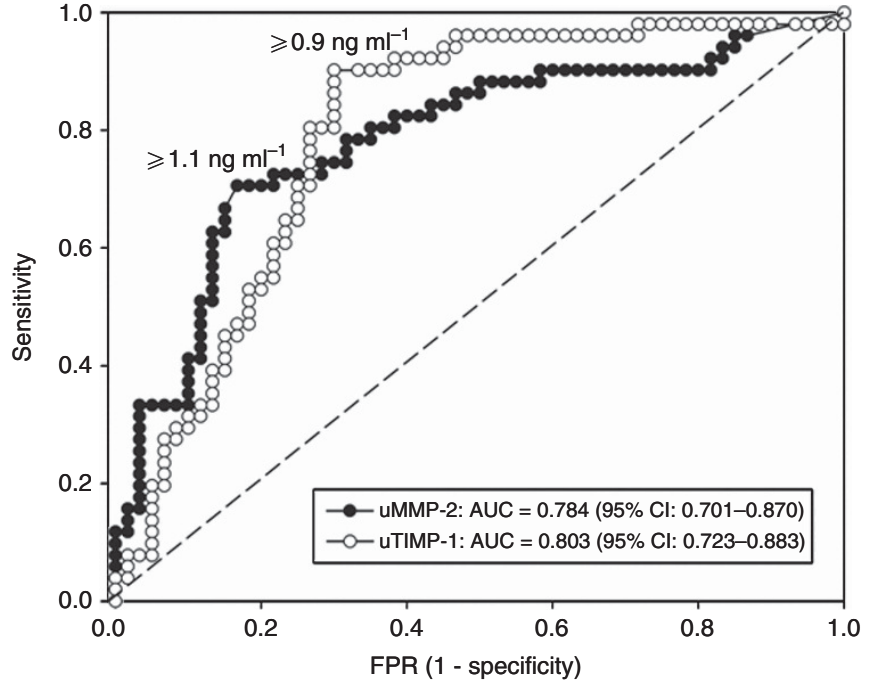

Figure 2. Receiver operating characteristic curves for urinary biomarkers. Receiver operating characteristic curves and optimal cutoff values for UMMP-2 and UTIMP-1 for differentiating between PDAC and controls. Dashed lines represent chance diagonal.

Table 2. Statistical performance characteristics of urinary biomarkers to differentiate PDAC from controls

\begin{tabular}{|l|c|c|c|c|}
\hline Biomarker & $\begin{array}{c}\text { Sensitivity } \\
(\%)\end{array}$ & $\begin{array}{c}\text { Specificity } \\
(\%)\end{array}$ & AUC & $95 \%$ Cl \\
\hline uMMP-2 $^{\text {a }}$ & 70 & 85 & 0.784 & $0.701-0.870$ \\
\hline uTIMP-1 $^{\text {a }}$ & 90 & 70 & 0.803 & $0.723-0.883$ \\
\hline uMMP-2 $^{\text {a }}$ uTIMP-1 $^{\text {a }}$ & 91 & 75 & 0.861 & $0.790-0.937$ \\
\hline
\end{tabular}

Abbreviations: $\mathrm{AUC}=$ area under the curve; $\mathrm{Cl}=$ confidence interval; $\mathrm{PDAC}=$ pancreatic ductal adenocarcinoma; $\mathrm{ROC}=$ receiver operating characteristic; $\mathrm{UMMP}-2=$ urinary matrix metalloprotease-2; UTIMP-1 = urinary tissue inhibitor of metalloprotease-1.

${ }^{\text {a }}$ Cutoff values for MMP-2 $\left(>1.1 \mathrm{ng} \mathrm{ml}^{-1}\right)$ and TIMP-1 $\left(>0.9 \mathrm{ng} \mathrm{ml}^{-1}\right)$ were identified using the Youden index in ROC analysis to compute sensitivity and specificity, respectively.

\begin{tabular}{|c|c|c|c|}
\hline $\begin{array}{l}\text { uMMP-2 } \\
\left(\geqslant 1.1 \mathrm{ng} \mathrm{ml}^{-1}\right)\end{array}$ & $\begin{array}{c}\text { uTIMP-1 } \\
\left(\geqslant 0.9 \mathrm{ng} \mathrm{ml}^{-1}\right)\end{array}$ & $\begin{array}{c}\text { Probability of } \\
\text { PDAC }\end{array}$ & $95 \% \mathrm{Cl}$ \\
\hline No & No & $8 \%$ & $3-18 \%$ \\
\hline Yes & No & $35 \%$ & $13-60 \%$ \\
\hline No & Yes & $50 \%$ & $32-67 \%$ \\
\hline Yes & Yes & $85 \%$ & $72-93 \%$ \\
\hline \multicolumn{4}{|c|}{$\begin{array}{l}\text { Abbreviations: } \quad \mathrm{Cl}=\text { confidence interval; } \quad \mathrm{PDAC}=\text { pancreatic ductal adenocarcinoma; } \\
\text { uMMP-2 = urinary matrix metalloprotease-2; UTIMP-1 }=\text { urinary tissue inhibitor of metallo- } \\
\text { protease-1. }\end{array}$} \\
\hline
\end{tabular}

ductal epithelium in the normal pancreas was completely devoid of TIMP-1 staining (Figures 3A and B), whereas neoplastic ducts in the cancer tissue had a marked increase in TIMP-1 staining (Figures 3C and D). Interestingly, PDAC-associated stroma was largely negative for TIMP-1 expression, which was limited to the ducts. When stratified according to a staining score (SS) range of $0-3$ arbitrary units (where $0=$ no expression, $3=$ very strong expression), mean TIMP-1 staining intensity was significantly higher in PDAC ( $\mathrm{SS}=1.90, n=44, P<0.00001)$ compared with normal pancreas $(\mathrm{SS}=0, n=16)$. In contrast, pNETs expressed
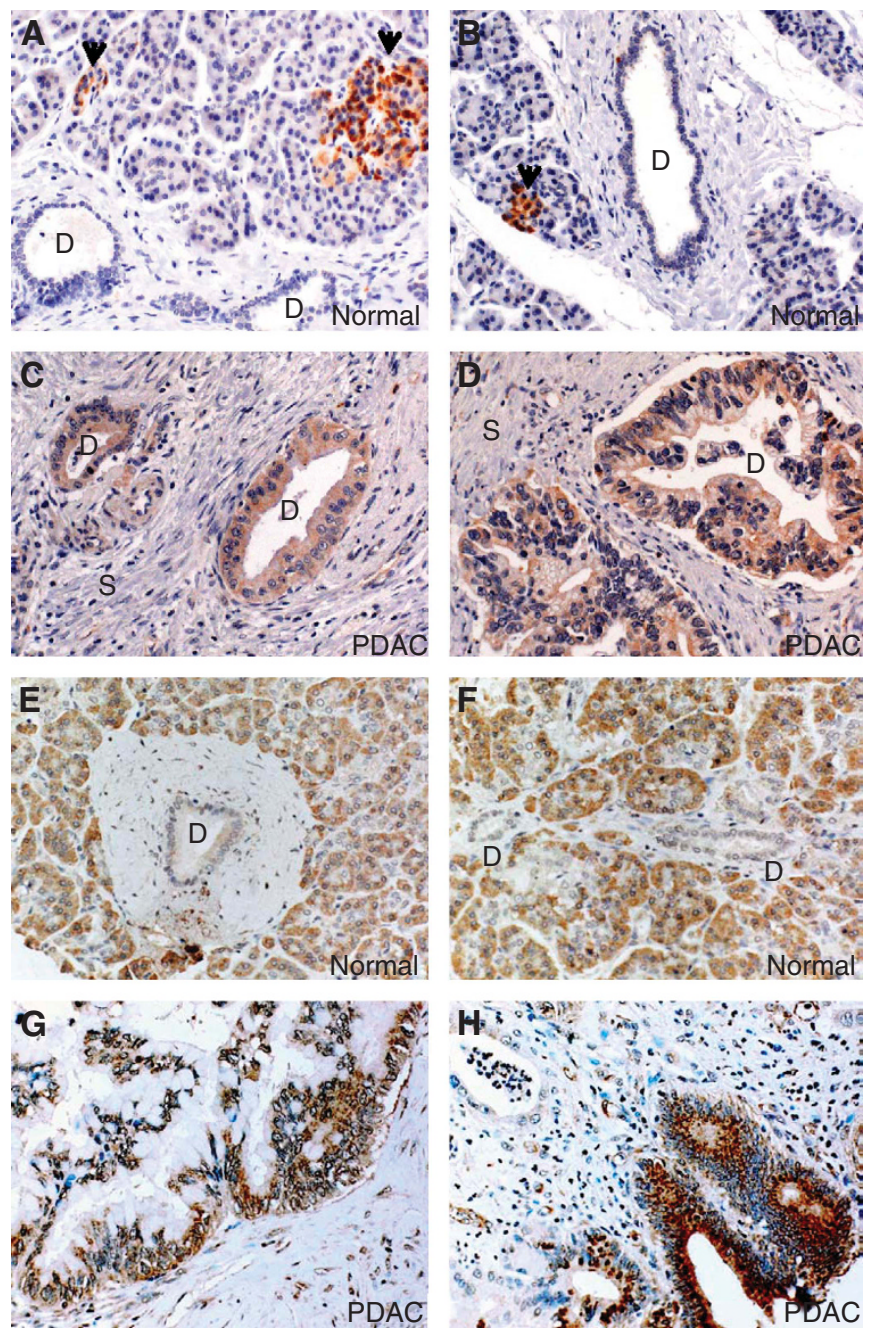

Figure 3. Matrix metalloprotease -2 and TIMP-1 expression is upregulated in human pancreatic tumour tissues. Immunohistochemistry analyses of TIMP-1 and MMP-2 in human pancreatic tumours and adjacent normal tissues. TIMP-1 protein expression was absent in normal pancreatic tissue (A and B), with the exception of islets, which stained focally positive, however, TIMP-1 expression was significantly upregulated in PDAC tissue (C and D). Moderate MMP-2 expression was detected in the normal pancreatic acinar tissues but not in the ductal epithelium (E and $\mathbf{F}$ ), whereas significantly higher MMP-2 expression was detected in pancreatic adenocarcinoma tissues ( $\mathbf{G}$ and $\mathbf{H}$ ).

very low or no TIMP-1 (25\% positive, $n=4$, SS $=0.25$, data not shown). Moderate levels of MMP-2 expression were detected in normal pancreatic acinar tissue $(n=8)$, however, the normal ductal epithelium was found to be largely negative for MMP-2 staining (Figures $3 \mathrm{E}$ and F). In contrast, in a majority $(\sim 87 \%)$ of the PDAC tissues $(n=44)$ strong MMP-2 expression was detected in the malignant ducts, with increased luminal staining in some instances (Figures $3 \mathrm{G}$ and $\mathrm{H}$ ).

\section{DISCUSSION}

In this study, we report for the first time that elevated levels of uMMP-2 and UTIMP-1 can identify the presence of pancreatic malignancies. Several distinct MMP species can be detected with significantly higher frequency in urine from pancreatic cancer patients compared with healthy controls. Of these, uMMP-2 but not uMMP-9 was a significant independent predictor of the 
presence of pancreatic cancer. We found that total uMMP-2 levels (via ELISA) as well as uMMP-2 activity (via substrate gel electrophoresis) were significantly higher in the pancreatic cancer groups compared with the controls. In addition, uMMP-2 was an independent predictor of the presence of neuroendocrine cancer (pNET). Urinary TIMP-1 levels were able to distinguish not only pancreatic cancer from control but also PDAC from pNET cancer groups.

Several studies have analysed the efficacy of serum MMP-9 and MMP-2 for the detection of pancreatic cancer (Mroczko et al, 2009; Brand et al, 2011). However, to date, very few studies have attempted to use urine as a source for biomarkers for pancreatic cancer (Nolen and Lokshin, 2011; Sorio et al, 2011). Our report is the first to measure urinary levels of the gelatinases MMP-2 and MMP-9 and their endogenous inhibitors TIMP-1 and TIMP-2 for the detection of pancreatic cancer. Compared with biomarker analyses in tissue specimens, the measurement of MMP-2 and TIMP-1 in urine of cancer patients provides several advantages. Analysis of urinary biomarkers is a truly non-invasive approach that does not require tumour biopsies or blood draws, and can be used for patients with both resectable and non-resectable disease. In addition, samples can be collected easily over time and used frequently to judge therapeutic efficacy. Our study indicates that uTIMP-1 alone (cutoff $>0.9 \mathrm{ng} \mathrm{m}^{-1}$ ) provides $90 \%$ sensitivity, $70 \%$ specificity and AUC of 0.803 , which is a marked improvement over the reported AUC of 0.716 for CA19-9 (Sandblom et al, 2008), the currently utilised biomarker for PDAC. In our study, AUC values improved to 0.861 for the combined detection of uTIMP-1 and uMMP-2 (sensitivity 91\%; specificity 75\%). Multivariable logistic regression confirmed that uMMP-2 $\geqslant 1.1 \mathrm{ng} \mathrm{ml}^{-1}$ (odds ratio $6.1,95 \%$ CI: $2.3-17.0$, likelihood ratio test $=12.3, P<0.001$ ) and uTIMP-1 $\geqslant 0.9 \mathrm{ng} \mathrm{ml}^{-1}$ (odds ratio 11.0, 95\% CI: $3.6-34.4$, likelihood ratio test $=20.4, P<0.001$ ) were significant independent predictive biomarkers of PDAC.

The gelatinases MMP-2 and MMP-9 are upregulated in PDAC and have been suggested to promote pancreatic cancer progression. Elevated levels of active MMP-2 and MMP-9 enzyme can be detected in exocrine pancreatic secretions (Yokoyama et al, 2002) as well as malignant ascites (Sun et al, 2003) of PDAC patients. In addition, two recent studies have implicated pancreatic stellate cellassociated MMP-2 in promoting pancreatic tumour progression and metastasis (Schneiderhan et al, 2007). However, the efficacy of using MMP-2 or MMP-9 as circulating biomarkers for PDAC has not been demonstrated. A study that analysed serum MMP-2 reported that levels did not significantly differ between PDAC, healthy controls or benign pancreatic disease (Brand et al, 2011). Our study represents the first to analyse uMMP-2 for the detection of pancreatic cancer. We found that uMMP-2 but not uMMP-9 levels were significantly higher in the pancreatic cancer groups compared with the controls. In addition, uMMP-2 was an independent predictor of the presence of pNET. Two recent studies have evaluated serum MMP-9 levels in correlation with PDAC with somewhat conflicting findings. Brand et al (2011) reported higher serum MMP-9 levels in PDAC patients compared with healthy controls, however, MMP-9 levels did not differ significantly between PDAC and benign pancreatic diseases. A second study indicated a potential clinical value for serum MMP-9 measurement as an independent prognostic factor for patient survival in PDAC (Mroczko et al, 2009). In contrast, in our study we found that uMMP-9 levels remained unchanged between the pancreatic cancer or control groups.

High TIMP-1 expression has been reported to be indicative of poor prognosis in both pancreatic and breast cancer (Bramhall et al, 1996; Nakopoulou et al, 2002). A number of gene expression or proteomic approaches have identified TIMP-1 as a candidate serum and tissue biomarker for human PDAC, as well as Krasdriven transgenic mouse model of pancreatic cancer in recent years
(Zhou et al, 1998; Faca et al, 2008). Consistent with these findings, TIMP-1 levels are significantly elevated in sera, (Zhou et al, 1998; Faca et al, 2008; Mroczko et al, 2009; Brand et al, 2011) exocrine pancreatic secretions and tumour tissue lysates (Lekstan et al, 2013) from PDAC patients compared with the controls. A recent study has reported the efficacy of utilising a combination of serum TIMP-1 and lipocalin 2 as a diagnostic test for the early detection of familial pancreatic cancer (Slater et al, 2013). However, in these previous studies TIMP-1 alone was not able to outperform the current clinically used PDAC marker, CA19-9, but TIMP-1 used in conjunction with a panel of proteins had higher efficacy (Zhou et al, 1998; Faca et al, 2008; Brand et al, 2011). In contrast, our data indicate that uTIMP-1 alone can differentiate between PDAC and control with $90 \%$ sensitivity and $70 \%$ specificity, and that this efficacy is improved when uMMP-2 detection is multiplexed with uTIMP-1 levels, resulting in sensitivity of $91 \%$ and specificity of $75 \%$. In addition, uTIMP-1 levels could also distinguish between PDAC and pNET in this study suggesting that TIMP-1 may have a specific role in the progression of exocrine pancreatic adenocarcinomas but not NETs. To date, there have been very few studies that have evaluated the contributions of MMPs and/or TIMPs in pNET progression or their usefulness as biomarkers for pNET. Our results indicate a novel correlation between elevated uMMP-2 levels and pNET. It remains to be seen whether combining uMMP2 analysis with serum CgA levels will improve accuracy for pNET detection. These studies are currently underway in our laboratory.

Finally, our IHC staining results appeared to be consistent with our finding of uMMP-2 and UTIMP-1 as urinary biomarkers of pancreatic cancer. Normal pancreatic tissues have low-to-moderate levels of MMP-2 staining with the majority of positive expression present in the acinar tissue. However, MMP-2 staining was significantly upregulated in neoplastic pancreatic ducts (Figures 3G and $\mathrm{H}$ ). Matrix metalloprotease-2 expression in normal acinar tissue appears to be mainly cytoplasmic (intracellular), whereas in PDAC tissues, MMP-2 localisation was mainly present in ductal and stromal cells. The difference in localisation of MMP-2 in normal $v s$ neoplastic pancreas (intracellular $v s$ secreted) may account for the increase in MMP-2 levels observed in urine from PDAC patients.

Active MMP-2 was detected in 91\% exocrine pancreatic secretion samples from PDAC patients compared with only $18 \%$ of those with chronic pancreatitis (Yokoyama et al, 2002). Several recent biomarker studies have shown that serum levels of TIMP-1 are significantly higher in PDAC compared with chronic pancreatitis (which was comparable to controls) and that serum TIMP-1 levels can distinguish PDAC from chronic pancreatitis as well as distinguish early-stage resectable PDAC cases from pancreatitis cases (Pan et al, 2011; Poruk et al, 2013). Taken together, these studies make it highly likely that a marker panel including uMMP-2 and UTIMP-1 may be able to distinguish pancreatic cancer from chronic pancreatitis.

Given the low incidence of pancreatic malignancies and the fact that a majority of cases are sporadic, screening for these malignancies within the general population is not recommended. In fact, the presence or level of these urinary biomarkers in the asymptomatic average-risk patient should be interpreted with caution and accompanied by further diagnostic workup. However, pancreatic cancer screening in high-risk groups (which account for $\sim 10 \%$ of total cases) may be beneficial. High-risk groups for PDAC comprises individuals with a family history of pancreatic cancer, hereditary pancreatitis, new onset ( $<4$ years), type 2 diabetes mellitus, obesity, Peutz-Jeghers disorder, cystic fibrosis, familial cancer syndromes such as Lynch syndrome, adenomatous polyposis (APC mutation), and hereditary breast and ovarian cancer syndrome (BRCA1 and BRCA2 mutations). Whereas an increased risk for developing pNET is associated with familial syndromes such as multiple endocrine neoplasia type 1, von 
Hippel-Lindau, tuberous sclerosis complex and neurofibromatosis type 1 . In fact, a family history of pancreatic cancer increases risk up to five-fold with a younger age of onset ( $<50$ years) among kindred of patients (Brune et al, 2010; Klein, 2011). Therefore, periodic screening for early disease using non-invasive methods within these high-risk populations could have a significant clinical impact on the detection, treatment and outcome of these deadly cancers. In the case of sporadic PDAC, a non-invasive test could prove useful in conjunction with currently used tests such as CA19-9 to improve therapeutic efficacy. Genetic analysis of PDAC has indicated that the time span for initiation of a pancreatic tumour followed by selection of a parental malignant clone and thereafter, acquisition of metastatic potential is of the order of approximately 15 years (Yachida et al, 2010). Therefore, there may exist a broader window of opportunity for early detection and prevention of this deadly disease. Although the clinical, epidemiological and statistical standards for sensitivity and specificity (as well as predictive values) depend heavily on the cohorts being evaluated, the evidence from our study suggests that developing a panel of predictive biomarkers would provide potential clinical usefulness for both diagnosis as well as screening and the next step would be a validation study to assess the generalisability of these results. The predictive biomarkers (uMMP-2 and uTIMP-1), which we identified as being independently able to differentiate between PDAC and controls in our study, have excellent combined sensitivity (91\%) and good specificity (75\%). The combined AUC also suggests excellent differential diagnosis and classification accuracy based on the statistically derived cutoffs for each of the two biomarkers. That said, these urinary biomarkers, particularly in combination, have performance characteristics that can be regarded as useful in differentiating pancreatic cancer patients from controls in the setting in which individuals are either classified as 'high risk' or in the setting where individuals undergo a clinical work-up where predictive biomarkers are included in conjunction with clinical, imaging and laboratory tests. The urinary biomarkers identified in our study may prove useful in the detection of pancreatic malignancies in high-risk populations at a stage when successful resection and therapy are possible. Further studies using appropriate patient cohorts will determine the efficacy of using these urinary biomarkers for screening high-risk populations for the presence of pancreatic malignancies.

\section{ACKNOWLEDGEMENTS}

This work is dedicated to the memory of our beloved mentor, the late M Judah Folkman, MD. The authors remain extremely grateful for his enthusiastic interest in this work and for many helpful discussions and suggestions. This work was supported by the Advanced Medical Research Foundation, NIH PO1 CA45548, NIH R01 CA118764, NIH grants R01 CA 151532-01A1 and 5P50CA 127003-5 (Gastrointestinal Cancer SPORE). We thank Kristin Johnson, Medical Graphics Core Director of the Vascular Biology Program for assistance with the figures.

\section{REFERENCES}

Ballehaninna U, Chamberlain R (2012) The clinical utility of serum CA 19-9 in the diagnosis, prognosis and management of pancreatic adenocarcinoma: an evidence based appraisal. J Gastrointest Oncol 3: 105-119.

Bramhall S, Stamp G, Dunn J, Lemoine N, Neoptolemos J (1996) Expression of collagenase (MMP2), stromelysin (MMP3) and tissue inhibitor of the metalloproteinases (TIMP1) in pancreatic and ampullary disease. Br J Cancer 73: 972-978.
Brand R, Nolen B, Zeh H, Allen P, Eloubeidi M, Goldberg M, Elton E, Arnoletti J, Christein J, Vickers S, Langmead C, Landsittel D, Whitcomb D, Grizzle W, Lokshin A (2011) Serum biomarker panels for the detection of pancreatic cancer. Clin Cancer Res 17: 805-816.

Brune K, Lau B, Palmisano E, Canto M, Goggins M, Hruban R, Klein A (2010) Importance of age of onset in pancreatic cancer kindreds. J Natl Cancer Inst 102: 119-126.

Canto M, Goggins M, Hruban R, Petersen G, Giardiello F, Yeo C, Fishman E, Brune K, Axilbund J, Griffin C, Ali S, Richman J, Jagannath S, Kantsevoy S, Kalloo A (2006) Screening for early pancreatic neoplasia in high-risk individuals: a prospective controlled study. Clin Gastroenterol Hepatol 4: 766-781quiz 665.

Crnogorac-Jurcevic T, Efthimiou E, Nielsen T, Loader J, Terris B, Stamp G, Baron A, Scarpa A, Lemoine N (2002) Expression profiling of microdissected pancreatic adenocarcinomas. Oncogene 21: 4587-4594.

Durlik M, Gardian K (2012) Metalloproteinase 2 and 9 activity in the development of pancreatic cancer. Pol Przegl Chir 84: 377-382.

Evans J, Ghaneh P, Kawesha A, Neoptolemos J (1997) Role of matrix metalloproteinases and their inhibitors in pancreatic cancer. Digestion 58: 520-528.

Faca V, Song K, Wang H, Zhang Q, Krasnoselsky A, Newcomb L, Plentz R, Gurumurthy S, Redston M, Pitteri S, Pereira-Faca S, Ireton R, Katayama H, Glukhova V, Phanstiel D, Brenner D, Anderson M, Misek D, Scholler N, Urban N, Barnett M, Edelstein C, Goodman G, Thornquist M, McIntosh M, DePinho R, Bardeesy N, Hanash S (2008) A mouse to human search for plasma proteome changes associated with pancreatic tumor development. PLoS Med 5: e123.

Goggins M (2005) Molecular markers of early pancreatic cancer. J Clin Oncol 23: $4524-4531$.

Goggins M (2007) Identifying molecular markers for the early detection of pancreatic neoplasia. Semin Oncol 34: 303-310.

Kaur S, Baine M, Jain M, Sasson A, Batra S (2012) Early diagnosis of pancreatic cancer: challenges and new developments. Biomark Med 6: $597-612$.

Klein A (2011) Genetic susceptibility to pancreatic cancer. Mol Carcinog 51: $14-24$.

Kulke M, Siu L, Tepper J, Fisher G, Jaffe D, Haller D, Ellis L, Benedetti J, Bergsland E, Hobday T, Van Cutsem E, Pingpank J, Oberg K, Cohen S, Posner M, Yao J (2011) Future directions in the treatment of neuroendocrine tumors: consensus report of the National Cancer Institute Neuroendocrine Tumor clinical trials planning meeting. J Clin Oncol 29: 934-943.

Lekstan A, Olakowski M, Jablonska B, Labuzek K, Olakowska E, Filip I, Lampe P (2013) Concentration of gelatinases and their tissue inhibitors in pancreatic inflammatory and neoplastic tumors and their influence on the early postoperative course. Pol Przegl Chir 85: 65-72.

Li D, Xie K, Wolff R, Abbruzzese J (2004) Pancreatic cancer. Lancet 363: 1049-1057.

Maitra A, Hruban R (2008) Pancreatic cancer. Annu Rev Pathol 3: 157-188.

Matsuyama Y, Takao S, Aikou T (2002) Comparison of matrix metalloproteinase expression between primary tumors with or without liver metastasis in pancreatic and colorectal carcinomas. J Surg Oncol 80: $105-110$.

Modlin IM, Gustafsson B, Moss S, Pavel M, Tsolakis A, Kidd M (2010) Chromogranin A-biological function and clinical utility in neuro endocrine tumor disease. Ann Surg Oncol 17: 2427-2443.

Modlin I, Oberg K, Chung D, Jensen R, de Herder W, Thakker R, Caplin M, Delle Fave G, Kaltsas G, Krenning E, Moss S, Nilsson O, Rindi G, Salazar R, Ruszniewski P, Sundin A (2008) Gastroenteropancreatic neuroendocrine tumours. Lancet Oncol 9: 61-72.

Moses M, Wiederschain D, Loughlin K, Zurakowski D, Lamb C, Freeman M (1998) Increased incidence of matrix metalloproteinases in urine of cancer patients. Cancer Res 58: 1395-1399.

Mroczko B, Lukaszewicz-Zajac M, Wereszczynska-Siemiatkowska U, Groblewska M, Gryko M, Kedra B, Jurkowska G, Szmitkowski M (2009) Clinical significance of the measurements of serum matrix metalloproteinase-9 and its inhibitor (tissue inhibitor of metalloproteinase-1) in patients with pancreatic cancer: metalloproteinase-9 as an independent prognostic factor. Pancreas 38: 613-618.

Nakopoulou L, Giannopoulou I, Stefanaki K, Panayotopoulou E, Tsirmpa I, Alexandrou P, Mavrommatis J, Katsarou S, Davaris P (2002) Enhanced mRNA expression of tissue inhibitor of metalloproteinase-1 (TIMP-1) in breast carcinomas is correlated with adverse prognosis. J Pathol 197: 307-313. 
Nolen B, Lokshin A (2011) The advancement of biomarker-based diagnostic tools for ovarian, breast, and pancreatic cancer through the use of urine as an analytical biofluid. Int J Biol Markers 26: 141-152.

Ozkan H, Kaya M, Cengiz A (2003) Comparison of tumor marker CA 242 with CA 19-9 and carcinoembryonic antigen (CEA) in pancreatic cancer. Hepatogastroenterology 50: 1669-1674.

Pan S, Chen R, Crispin D, May D, Stevens T, McIntosh M, Bronner M, Ziogas A, Anton-Culver H, Brentnall T (2011) Protein alterations associated with pancreatic cancer and chronic pancreatitis found in human plasma using global quantitative proteomics profiling. J Proteome Res 10: 2359-2376.

Plockinger U, Rindi G, Arnold R, Eriksson B, Krenning E, de Herder W, Goede A, Caplin M, Oberg K, Reubi J, Nilsson O, Delle Fave G, Ruszniewski P, Ahlman H, Wiedenmann B (2004) Guidelines for the diagnosis and treatment of neuroendocrine gastrointestinal tumours. A consensus statement on behalf of the European Neuroendocrine Tumour Society (ENETS). Neuroendocrinology 80: 394-424.

Poley J, Kluijt I, Gouma D, Harinck F, Wagner A, Aalfs C, van Eijck C, Cats A, Kuipers E, Nio Y, Fockens P, Bruno M (2009) The yield of first-time endoscopic ultrasonography in screening individuals at a high risk of developing pancreatic cancer. Am J Gastroenterol 104: 2175-2181.

Pories S, Zurakowski D, Roy R, Lamb C, Raza S, Exarhopoulos A, Scheib R, Schumer S, Lenahan C, Borges V, Louis G, Anand A, Isakovich N, Hirshfield-Bartek J, Wewer U, Lotz M, Moses M (2008) Urinary metalloproteinases: noninvasive biomarkers for breast cancer risk assessment. Cancer Epidemiol Biomarkers Prev 17: 1034-1042.

Poruk K, Firpo M, Scaife C, Adler D, Emerson L, Boucher K, Mulvihill S (2013) Serum osteopontin and tissue inhibitor of metalloproteinase 1 as diagnostic and prognostic biomarkers for pancreatic adenocarcinoma. Pancreas 42: 193-197.

Roy R, Coticchia C, Yang J, Moses M (2010) Biomarkers in cancer biology. In: Biomarkers of Toxicity: A New Era in Medicine, Vaidya V, Bonaventure J (eds), pp 355-380. John Wiley and Sons: NY, USA.

Roy R, Louis G, Loughlin K, Wiederschain D, Kilroy S, Lamb C, Zurakowski D, Moses M (2008) Tumor-specific urinary matrix metalloproteinase fingerprinting: identification of high molecular weight urinary matrix metalloproteinase species. Clin Cancer Res 14: 6610-6617.

Roy R, Rodig S, Bielenberg D, Zurakowski D, Moses M (2011) ADAM12 transmembrane and secreted isoforms promote breast tumor growth: a distinct role for ADAM12-S protein in tumor metastasis. J Biol Chem 286: 20758-20768.

Roy R, Wewer U, Zurakowski D, Pories S, Moses M (2004) ADAM 12 cleaves extracellular matrix proteins and correlates with cancer status and stage. J Biol Chem 279: 51323-51330.

Sandblom G, Granroth S, Rasmussen I (2008) TPS, CA 19-9, VEGF-A, and $\mathrm{CEA}$ as diagnostic and prognostic factors in patients with mass lesions in the pancreatic head. Ups J Med Sci 113: 57-64.

Schneiderhan W, Diaz F, Fundel M, Zhou S, Siech M, Hasel C, Moller P, Gschwend J, Seufferlein T, Gress T, Adler G, Bachem M (2007) Pancreatic stellate cells are an important source of MMP-2 in human pancreatic cancer and accelerate tumor progression in a murine xenograft model and CAM assay. J Cell Sci 120: 512-519.

Seregni E, Ferrari L, Bajetta E, Martinetti A, Bombardieri E (2001) Clinical significance of blood chromogranin A measurement in neuroendocrine tumours. Ann Oncol 12(Suppl 2): S69-S72.

Slater E, Fendrich V, Strauch K, Rospleszcz S, Ramaswamy A, Matthai E, Chaloupka B, Gress T, Langer P, Bartsch D (2013) LCN2 and TIMP1 as potential serum markers for the early detection of familial pancreatic cancer. Transl Oncol 6: 99-103.

Smith E, Manfredi M, Scott R, Black P, Moses M (2007) A recurrent craniopharyngioma illustrates the potential usefulness of urinary matrix metalloproteinases as noninvasive biomarkers: case report. Neurosurgery 60: E1148-E1149discussion E1149.
Smith E, Zurakowski D, Saad A, Scott R, Moses MA (2008) Urinary biomarkers predict brain tumor presence and response to therapy. Clin Cancer Res 14: 2378-2386.

Sorio C, Mafficini A, Furlan F, Barbi S, Bonora A, Brocco G, Blasi F, Talamini G, Bassi C, Scarpa A (2011) Elevated urinary levels of urokinase-type plasminogen activator receptor (uPAR) in pancreatic ductal adenocarcinoma identify a clinically high-risk group. BMC Cancer 11: 448.

Steinberg W (1990) The clinical utility of the CA 19-9 tumor-associated antigen. Am J Gastroenterol 85: 350-355.

Sun C, Rosendahl A, Ansari D, Andersson R (2011) Proteome-based biomarkers in pancreatic cancer. World J Gastroenterol 17: 4845-4852.

Sun X, Dong W, Yu B, Luo H, Yu J (2003) Detection of type IV collagenase activity in malignant ascites. World J Gastroenterol 9: 2592-2595.

Tempero M, Uchida E, Takasaki H, Burnett D, Steplewski Z, Pour P (1987) Relationship of carbohydrate antigen 19-9 and Lewis antigens in pancreatic cancer. Cancer Res 47: 5501-5503.

Ter-Minassian M, Chan J, Hooshmand S, Brais L, Daskalova A, Heafield R, Buchanan L, Qian Z, Fuchs C, Lin X, Christiani D, Kulke M (2013) Clinical presentation, recurrence, and survival in patients with neuroendocrine tumors: results from a prospective institutional database. Endocr Relat Cancer 20: 187-196.

Winter J, Yeo C, Brody J (2012) Diagnostic, prognostic, and predictive biomarkers in pancreatic cancer. J Surg Oncol 107: 15-22.

Yachida S, Jones S, Bozic I, Antal T, Leary R, Fu B, Kamiyama M, Hruban RH, Eshleman JR, Nowak MA et al. (2010) Distant metastasis occurs late during the genetic evolution of pancreatic cancer. Nature 467: 1114-1117.

Yang X, Staren E, Howard J, Iwamura T, Bartsch J, Appert H (2001) Invasiveness and MMP expression in pancreatic carcinoma. J Surg Res 98: 33-39.

Yao J, Hassan M, Phan A, Dagohoy C, Leary C, Mares J, Abdalla E, Fleming J, Vauthey J, Rashid A, Evans D (2008) One hundred years after "carcinoid": epidemiology of and prognostic factors for neuroendocrine tumors in 35,825 cases in the United States. J Clin Oncol 26: 3063-3072.

Yao J, Pavel M, Phan A, Kulke M, Hoosen St S, Peter J, Cherfi A, Oberg K (2011) Chromogranin A and neuron-specific enolase as prognostic markers in patients with advanced pNET treated with everolimus. J Clin Endocrinol Metab 96: 3741-3749.

Yeo T, Hruban R, Leach S, Wilentz R, Sohn T, Kern S, Iacobuzio-Donahue C, Maitra A, Goggins M, Canto M, Abrams R, Laheru D, Jaffee E, Hidalgo M, Yeo CJ (2002) Pancreatic cancer. Curr Probl Cancer 26: $176-275$.

Yokoyama M, Ochi K, Ichimura M, Mizushima T, Shinji T, Koide N, Tsurumi T, Hasuoka H, Harada M (2002) Matrix metalloproteinase-2 in pancreatic juice for diagnosis of pancreatic cancer. Pancreas 24: 344-347.

Zatelli M, Torta M, Leon A, Ambrosio M, Gion M, Tomassetti P, De Braud F, Delle Fave G, Dogliotti L, degli Uberti E (2007) Chromogranin A as a marker of neuroendocrine neoplasia: an Italian Multicenter Study. Endocr Relat Cancer 14: 473-482.

Zhou W, Sokoll L, Bruzek D, Zhang L, Velculescu V, Goldin S, Hruban R, Kern S, Hamilton S, Chan D, Vogelstein B, Kinzler K (1998) Identifying markers for pancreatic cancer by gene expression analysis. Cancer Epidemiol Biomarkers Prev 7: 109-112.

Zhou X, Obuchowski N, McClish D (2011) Statistical Methods in Diagnostic Medicine, 2nd edn, 13-55. John Wiley: New York.

This work is published under the standard license to publish agreement. After 12 months the work will become freely available and the license terms will switch to a Creative Commons AttributionNonCommercial-Share Alike 3.0 Unported License. 